\title{
ARTIGOS DE REVISÃO \\ MEMÓRIA ORGANIZACIONAL, GESTÃO DO CONHECIMENTO E COMPORTAMENTOS DE CIDADANIA ORGANIZACIONAL
}

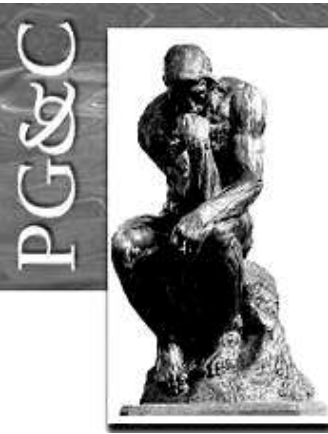

\author{
Paula Costa Neves \\ Doutora em Psicologia das Organizações pela Universidade de Coimbra, \\ Portugal. Professora do Instituto Politécnico de Coimbra, Escola \\ Superior de Educação, Portugal. \\ E-mail:pneves@esec.pt \\ José Pedro Cerdeira \\ Doutor em Psicologia Social pela Universidade de Coimbra, Portugal. \\ Professor do Instituto Politécnico de Coimbra, Escola Superior de \\ Educação, Portugal. \\ E-mail: jpcerd@esec.pt
}

\begin{abstract}
Resumo
Para obterem vantagens competitivas e assim realizarem a sua missão, as empresas e as organizações precisam de criar, usar e preservar uma memória dos conhecimentos acumulados no histórico da organização e nos processos organizacionais. Neste texto, utilizando uma metodologia de revisão de literatura de natureza narrativa, aborda-se o conceito de memória organizacional (MO), enquanto conhecimento organizacional que integra experiências passadas, arquivadas e vividas no contexto das organizações. Chama-se a atenção para a importância de preservar esta memória e para a necessidade de partilhar e manter o conhecimento dentro da organização, independentemente da rotatividade dos seus elementos. Evidencia-se a importância da partilha de conhecimento na construção e preservação da memória organizacional, destacando-se o papel desempenhado por duas variáveis essenciais: os comportamentos de cidadania organizacional e a confiança. Sugerem-se ainda algumas orientações estratégicas para a promoção de boas práticas de gestão do conhecimento e de construção da memória nas organizações.
\end{abstract}

Palavras-chave: Memória Organizacional. Gestão do Conhecimento. Comportamentos de Cidadania Organizacional. Partilha de Conhecimento. Confiança.

\section{ORGANIZATIONAL MEMORY, KNOWLEDGE MANAGEMENT AND ORGANIZATIONAL CITIZENSHIP BEHAVIORS}

\begin{abstract}
In order to obtain competitive advantage and thus fulfill their mission, companies and organizations need to create, use and preserve a memory of accumulated knowledge in the organization's history and organizational processes. This paper, using methodology narrative review deals with the concept of organizational memory (OM), as organizational knowledge that integrates past experiences, archived and lived by the people in the contexts of the organizations, and draws attention to the importance of preserving this memory, and to the need to share and maintain knowledge within the organization although turnover. In this context, the importance of knowledge sharing in the construction and preservation of organizational memory is highlighted, especially the role played by two essential variables: organizational citizenship behaviors and trust. Some strategic guidelines are also suggested for promotion of good practice of knowledge management and building organizational memory
\end{abstract}

Perspectivas em Gestão \& Conhecimento, João Pessoa, v. 8, n. 1, p. 3-19, jan./abr. 2018. DOI: http://dx.doi.org/10.21714/2236-417X2018v8n1p3.

http://periodicos.ufpb.br/ojs2/index.php/pgc. ISSN: 2236-417X. Publicação sob Licença (cc) EY-Nc-ND 
Keywords: Organizational Memory. Knowledge Management. Organizational Citizenship Behavior. Knowledge Sharing. Trust.

\section{INTRODUÇÃO}

Num ambiente político, social e económico turbulento e em contínua mudança, em que as empresas e as organizações precisam de obter ganhos de eficácia nos processos organizacionais para melhor competirem nos mercados globalizados, a partilha de informações e conhecimentos relevantes, adquiridos pelos colaboradores a partir da experiência acumulada, é crucial e não pode deixar de estar no centro das preocupações estratégias dos gestores. No entanto, não é fácil promover esta partilha, nem mesmo com recurso a sistemas tecnológicos mais ou menos sofisticados e dispendiosos, já que a decisão de o fazer é uma decisão pessoal e voluntária da parte de quem o construiu, o possui e o guarda.

O conhecimento individual dos membros de uma organização sobre a natureza das tarefas e dos processos organizacionais é muitas vezes informal e difícil de sistematizar. Baseia-se predominantemente nas aprendizagens e nas experiências idiossincráticas vividas pelos atores organizacionais, pelo que é um conhecimento espontâneo e subjectivamente organizado. Uma vez que é também um conhecimento com um valor instrumental significativo, tende a ser preservado para ser usado com o objetivo de facilitar o desempenho de tarefas na organização, para gerir carreiras, para satisfazer necessidades de obtenção de uma posição relevante nos grupos de trabalho ou para gerir a participação nos mais diversos processos na própria organização. No entanto, para além de ser um conhecimento útil para aqueles que o adquiriram, é também um conhecimento muito importante para a organização, pelo que não deve ser perdido, bem pelo contrário, é um conhecimento que deve ser organizado na memória da organização e partilhado com frequência e sempre que necessário.

Neste artigo, apresenta-se uma revisão da literatura utilizando uma metodologia de cariz narrativo realizada com o objetivo de realçar a importância da partilha de conhecimento organizacional para a construção e preservação da memória das organizações, com especial destaque para o papel desempenhado por duas variáveis essenciais: os comportamentos de cidadania organizacional e a confiança. Os textos foram selecionados em função do reconhecimento da pertinência dos argumentos dos autores para sustentar a defesa do ponto de vista acima exposto: o fortalecimento da confiança e a promoção de comportamentos de cidadania organizacional podem fomentar a partilha da memória organizacional.

\section{MEMÓRIA ORgANIZACIONAL}

Não há consenso sobre a definição do conceito de memória organizacional (MO), nem mesmo sobre a realidade designada pelo mesmo ou até sobre o nível em que esta pode ou não reconhecer-se nas práticas das organizações, ou seja, se o fenómeno deve ser entendido no plano dos indivíduos e dos grupos ou ao nível dos processos organizacionais ou tão só ao nível das metáforas organizacionais (úteis para a compreensão de algumas especificidades das organizações).

Stein (1995) entende que a memória organizacional se refere aos meios pelos quais se busca o conhecimento sobre a experiência do passado para o incorporar e dar suporte às atividades do presente, com o objetivo último de produzir maiores ganhos de eficácia organizacional, ou seja, a memória de uma organização é entendida como um sistema instrumental de gestão de informação, baseado na aquisição, conservação, manutenção, pesquisa e recuperação de informações relevantes para a gestão dos processos organizacionais. Van Heijst, Van Der Spek e Kruizinga (1997, p. 41), no entanto, definem o 
conceito como "uma representação explícita, desencarnada e persistente do conhecimento e da informação numa organização" reduzindo-a apenas ao conhecimento explícito e codificado das organizações, enquanto Huang, Fan, Chern e Yen (2013) se referem a ela como um repositório de conhecimentos e know-how de um conjunto de indivíduos que trabalham num determinado negócio, colocando-se numa perspetiva de capital humano. Neste sentido também, Nascimento et al.(2016) avançam com o argumento que a gestão da informação nas organizações e a gestão do conhecimento organizacional são ferramentas essenciais para a construção e preservação da memória organizacional e para a obtenção de vantagens competitivas em ambientes de mudança.

Neste texto situar-nos-emos em sintomia com a posição original de Nilakanta, Miller e Zhu (2006), assumindo também o entendimento da MO enquanto conhecimento organizacional que integra experiências passadas, arquivadas e vividas pelos seus elementos nos seus contextos técnicos, sociais e funcionais do ambiente de trabalho. No essencial, esta posição retoma a perspetiva proposta por Walsh e Ungson (1991), segundo a qual a MO se refere à capacidade de obter, reutilizar e recordar informações sobre as decisões anteriormente tomadas e os problemas já resolvidos. A MO é assim conceptualizada como a totalidade do conhecimento criado, valorizado e acumulado a partir das experiências passadas, que está na organização e que é utilizado para tomar as decisões no dia-a-dia. Estas memórias (conhecimentos) encontram-se armazenadas em diferentes repositórios ou recetáculos individuais (nos atuais e nos antigos elementos da organização) ou supraindividuais (na cultura, na estrutura, nos processos/ transformações/ rotinas, na ecologia dos espaços e em arquivos externos).

Uma vez que o conhecimento é armazenado em diferentes repositórios, o acesso aos mesmos pode estar mais ou menos condicionado pelos termos em que foi armazenado. Por exemplo, se o processo de recolha, organização e armazenamento foi protagonizado por indivíduos (que o fizeram voluntariamente, pelos seus próprios meios e a título pessoal) então a disponibilidade deste conhecimento depende sobretudo da sua vontade, o que quer dizer que a sua disponibilização a terceiros pode estar comprometida sempre que os curadores deste conhecimento abandonarem a organização ou sempre que estes alterarem as suas disposições para a partilha voluntária. O conhecimento e a MO podem assim perder algumas das suas componentes mais importantes com, por exemplo, a reforma de um colaborador, onde um "mundo" de memórias pode perder-se apenas pela mudança da empresa subcontratada para um determinado setor (SCHREIBER, 2015).

Outra forma de olhar para a memória organizacional, também do ponto de vista da retenção e preservação do conhecimento individual, é através do conceito de memória transativa. Wegner (1986) introduz o termo para caracterizar a especialização que ocorre entre indivíduos com relações interpessoais próximas, onde os indivíduos utilizam os conhecimentos uns dos outros enquanto extensões e ajudas externas da sua própria memória. $\mathrm{Na}$ medida em que cada um se responsabiliza por reter e preservar a memória específica de um conjunto de conhecimentos relativos a experiências particulares, torna-se possível a especialização na retenção e a complementaridade na evocação das memórias organizacionais dos diferentes colaboradores, o que amplia enormemente o potencial dos recursos de gestão do conhecimento da organização. Conhecido como "quem sabe o quê" nas organizações, a memória transativa faculta aos membros de uma organização o acesso a volumes de informação consideráveis, que vão muito para além da informação que possuem individualmente, e que se torna possível através da consciência intersubjetiva do somatório do conhecimento armazenado. A memória transativa tem duas componentes: o conhecimento específico que está na posse de cada um dos indivíduos e o conhecimento que pode ser obtido pelos processos de transação entre diferentes indivíduos, o qual permite a coordenação dos

Perspectivas em Gestão \& Conhecimento, João Pessoa, v. 8, n. 1, p. 3-19, jan./abr. 2018. 
conhecimentos e competências mútuas entre as partes envolvidas (WEGNER; GUILIANO; HERTEL, 1985).

A memória transativa é deste modo conceptualizada como propriedade de uma rede, cuja malha é representada pelas memórias individuais, que permite à organização gerir a distribuição das tarefas pelos colaboradores mais qualificados. Este entendimento do potencial da gestão da MO reveste-se da maior importância, principalmente na abordagem a tarefas não rotineiras e na resolução de novos problemas em contexto de incerteza ou de turbulência do ambiente organizacional (REN; CARLEY; ARGOTE, 2006). Na prática trata-se de uma divisão cognitiva do trabalho, em que os membros da organização se especializaram ao longo do tempo, acumulando e retendo conhecimentos em áreas diferenciadas, as quais numa dada situação podem ser convocados para resolver problemas específicos com implicações positivas na melhoria do desempenho das equipas de trabalho (REN; ARGOTE, 2011) e na obtenção de ganhos de eficácia e de vantagens competitivas relevantes.

No entanto, sendo também uma memória que mobiliza conhecimentos pessoais que podem ser percebidos como "propriedade" de quem os acumulou e preservou, a rotatividade tem um efeito disruptivo, podendo a memória ficar muito afetada quando os elementos abandonam a organização (ARGOTE; GUO, 2016).

A memória de uma organização é uma espécie de mapa com grandes quantidades de informação sobre experiências passadas, cuja mobilização e utilização permite à organização evitar cometer os mesmos erros, assegurando-se ao mesmo tempo que continua a usar as melhores práticas e a sabedoria coletiva (NILAKANTA; MILLER; ZHU, 2006). Assim, considerando que uma das funções mais importantes da MO é a de preservar e melhorar a competitividade, o desempenho (AMINU; MAHMOOD, 2016) e ajudar o desenvolvimento das organizações (MOLINA; VALENTIM, 2015) importa então abordar a forma como a MO pode ser preservada, com o objetivo de permitir em pleno o cumprimento desta sua função.

\subsection{Memória Organizacional e Gestão do Conhecimento}

O atual contexto de mudanças contínuas no ambiente social, cultural e económico, coloca às organizações o desafio de, permanentemente, gerirem a mudança organizacional. No entanto, em paralelo, as organizações necessitam de ser capazes de preservar as suas competências-chave, as suas capacidades, a estabilidade das relações com os seus stakeholders, a experiência acumulada, as práticas e o expertise - independentemente da rotatividade dos seus membros. A realização deste objetivo requer a promoção de uma cultura organizacional capaz de evidenciar claramente a importância e a relevância estratégica de gerar e preservar sistemática, contínua, e fidedignamente um acervo de memórias (conhecimentos) que sirva de base à tomada de decisão e à evidenciação permanente da missão e visão da organização (NASCIMENTO et al., 2016), ou seja, implica intervenções intencionais, orientadas para a criação e manutenção de repositórios com dados e informações pertinentes e a sua disponibilização a quem deles necessita (MOLINA; VALENTIM, 2015), mas também intervenções orientadas para a manutenção do conhecimento crítico na empresa ao longo do tempo.

A construção de repositórios com informação e conhecimento, ou seja a ação intencional de preservar conhecimento na MO pode vir a ser o elemento diferenciador no processo de tomada de decisão inteligente (SANTARÉM; VITORIANO, 2016), contribuindo assim para a obtenção de vantagens competitivas das empresas.

Não é fácil definir o que é conhecimento devido à sua natureza intangível, além de que este é muitas vezes utilizado enquanto sinónimo de informação e esta por vezes confundida com a noção de dados. Cardoso, Duarte Gomes e Rebelo (2003) clarificam cada um destes conceitos evidenciando a articulação entre os mesmos. Os conceitos são independentes, mas é

Perspectivas em Gestão \& Conhecimento, João Pessoa, v. 8, n. 1, p. 3-19, jan./abr. 2018. 
possível encontrar uma sequência evolutiva contínua entre dados (definidos enquanto elementos providos de significado, simples observações), informação (resultante da atribuição de propósitos e relevância aos dados) e o conhecimento em si que reflete as interpretações, reflexões e síntese dos dados e da informação. Assim é possível verificar que apenas as pessoas podem gerar conhecimento, embora nos repositórios das organizações (bases de dados, relatórios, etc.) possamos encontrar também dados e informações.

Para Nonaka e Takeuchi (1995) há dois tipos de conhecimento: o tácito e o explícito. 0 conhecimento tácito diz respeito ao know-how e à capacidade dos trabalhadores realizarem as suas tarefas no dia-a-dia. É um conhecimento pessoal, não formalizado, composto por modelos mentais, crenças, aptidões técnicas, indissociáveis do sujeito que as detém e do seu contexto, ou seja, é um conhecimento que é pertença do individuo e não da organização. É um tipo de conhecimento que pode conferir vantagem competitiva à organização por ser dificilmente difundido para o exterior e imitável por organizações concorrentes. 0 conhecimento explícito é aquele que está codificado, que é transmissível em linguagem formal e sistemática e que pode ser encontrado nos diferentes procedimentos, nas bases de dados, é portanto um conhecimento que está fora dos indivíduos e que é pertença da organização.

Embora se possa reconhecer que as organizações existem para além das pessoas que as integram, é necessário ganhar consciência que são as pessoas que obtêm informações, que resolvem problemas, que aprendem com a experiência, que a organizam e que tomam decisões no seu seio, modelando-a e dando-lhe um sentido. São também as pessoas que, através dos seus processos cognitivos, constroem o conhecimento, pelo que, uma parte importante do conhecimento (ou memória) organizacional, estando na posse dos indivíduos, pode perder-se com a saída dos elementos da organização, ou simplesmente pela transferência de uma liderança interna (PEREIRA et al., 2016). Se este conhecimento não tiver sido anteriormente disponibilizado, partilhado e transferido para outros membros da organização, ou se não tiver sido transformado em conhecimento organizacional, quando as pessoas abandonam a organização deixam um espaço livre na sua rede de relações e interações sociais, que pode ser entendido como uma lacuna (uma falta ou uma espécie de "buraco"), já que ao saírem as pessoas levam consigo as informações, os conhecimentos e a experiência acumulada ao longo de muitos anos, que com frequência mais ninguém possui.

A necessidade de preservar o conhecimento (ou memória), mantendo-o na organização, justifica a importância das organizações incentivarem a implementação de sistemas de gestão do conhecimento onde se incluem os processos de transferência. A transferência do conhecimento envolve a partilha do conhecimento pela fonte do conhecimento e a aquisição e utilização do conhecimento pelo recetor. Na sequência desta exposição centrar-nos-emos apenas na fonte, ou seja nos processos psicológicos que podem incentivar a partilha do conhecimento pessoal que cada um possui da memória organizacional.

A partilha do conhecimento refere-se à ação de difusão de informação relevante entre os elementos da organização, com o objetivo de contribuir para o conhecimento coletivo da organização e transferir valor e recursos para a organização (BARTOL; SRIVASTAVA, 2002), tendo sido relacionada com o desenvolvimento de inovações (SOUSA; AMARAL, 2012). Referese ainda ao grau em que os colaboradores transferem entre si o conhecimento adquirido (TEH; SUN, 2011) e ao respetivo impacto no desempenho individual (MARQUES; CARDOSO; ZAPPALÁ, 2008) e organizacional (BARTOL; SRIVASTAVA, 2002). A partilha do conhecimento é por estas duas ordens de razões um processo complexo, o qual se enquadra no contexto global de gestão do conhecimento organizacional, que para Bock e Kim (2002) representa a parte mais importante de toda a gestão do conhecimento.

A gestão do conhecimento, que tem sido apontada como um recurso da gestão das organizações para manter a sua competitividade (DAVENPORT; PRUSAK, 1998), é um processo que promove o fluxo do conhecimento entre indivíduos e grupos na organização, podendo ser 
descrito por uma sequência de quatro grandes etapas essenciais: aquisição, armazenamento distribuição e utilização do conhecimento. Concretamente a gestão do conhecimento inclui o desenvolvimento articulado de metodologias, ferramentas, técnicas e valores organizacionais para promover o fluxo de conhecimento entre os indivíduos e a recuperação, transformação e utilização deste conhecimento em atividades de melhoria e de inovação. Gerir o conhecimento numa organização permite recolher o que os elementos coletivamente sabem, aprenderam e se lembram do que correu bem, do que não resultou, das lições aprendidas e a sua reutilização nas práticas diárias e futuras, nomeadamente nos processos de tomada de decisão ou de desenvolvimento de novos projetos.

Nonaka e Takeuchi (1995) propõem um modelo de criação de conhecimento organizacional, conhecido como a "espiral do conhecimento" assente na interação dinâmica entre pessoas. Para estes autores a criação de conhecimento tem duas dimensões: uma epistemológica que se refere à distinção entre conhecimento tácito e explicito e uma dimensão ontológica que diz respeito aos níveis em que o conhecimento é criado (individual, grupal, organizacional ou interorganizacional) e processa-se através da interação dinâmica dos indivíduos na conversão do conhecimento tácito (individual) em conhecimento explícito, e este novamente em tácito, através dos quatro modos de conversão: socialização (de tácito para tácito); exteriorização (tácito para explícito), internalização (de explícito para tácito) e combinação (de tácito para explícito). Estes processos dinâmicos, que constituem o centro de criação de conhecimento organizacional, têm o seu núcleo na interação, ou seja o conhecimento é criado pela interação entre os indivíduos ou entre estes e os seus ambientes.

Os esforços de preservação do conhecimento existente na organização numa $\mathrm{MO}$, implica em primeiro lugar a referenciação do conhecimento relevante e a identificação da sua localização na organização. Não é possivel, criar mecanismos de obtenção e armazenamento de conhecimento se este não estiver localizado e identificado, tarefa que constiui um dos primeiros desafios da gestão do conhecimento. Muito frequentemente as organizações não têm informação acerca da natureza e localização do conhecimento que os seus colaboradores possuem, o que dificulta naturalmente a sua obtenção e utlização. Para Davenport e Prusak (1998), a inexistência de um mapeamento dos conhecimentos relevantes, a assimetria (concentração do conhecimento em determinados pontos da organziação, que seriam relevantes para outros departamentos) e a localização não identificada do conhecimento são obstáculos que dificultam a construção de uma MO que se pretende acessível a todos os elementos que em cada momento necessitam de a evocar, para suportar as tomadas de decisão que se pretendem inteligentes (SANTARÉM; VITORIANO, 2016).

Esta dificuldade no desenvolvimento de uma estrutura formal de acumulação, retenção e disponibilização fácil do conhecimento a todos os elementos, remete a procura e recolha de informação e conhecimento relevante em cada momento para as interações pessoais formais (reuniões) ou informais (redes de afinidade) ou ainda para as relações de proximidade. O conhecimento é "comprado" (na termionologia de Davenport e Prusak, 1998) à pessoa que está fisicamente próxima, em vez de se tentar saber quem na organização tem conhecimento mais relevante na matéria.

Os elevados custos para encontrar o conhecimento relevante em cada situação, são um dos maiores constragimentos na gestão do conhecimento nas organizações e constituem mesmo o seu maior desafio (CASCÃO, 2014). Identificar quem sabe o quê é uma tarefa morosa, pelo que se justifica a necessidade de possuir sistemas de gestão do conhecimento que permitam identificar a aceder às competências dos colaboradores.

O processo de armazenamento e distribuição da informação e de conhecimento entre os elementos da organização pode ser desenvolvido apenas com recurso a tecnologias de informação ou pode desenvolver-se pela promoção de práticas de interação entre os diferentes elementos da organização, intermediado ou não pelas tecnologias de informação e 
comunicação. Já a codificação do conhecimento, colocando-o ao alcance de toda a organização, é uma atividade que apenas pode ser realizada pelas pessoas. Não basta que as organizações tenham tecnologias de armazenamento de dados e repositórios de informação e conhecimento, é necessário que as pessoas, que dela fazem parte, partilhem por vontade própria o seu conhecimento, de modo a evidenciarem o seu valor e significado para os diferentes processos organizacionais.

No caso específico do conhecimento tácito, para que haja partilha de conhecimento, é indispensável que exista uma interação entre a fonte e o recetor (mediada, ou não, por tecnologias de informação) e uma disponibilidade do emissor para partilhar. O conhecimento tácito resulta de um processo de aprendizagem individual, frequentemente moroso e que pode exigir da organização um grande investimento em tempo e formação específica, mas que pode também permitir à organização manter a sua competitividade, uma vez que é um tipo de conhecimento que não é facilmente imitável ou transferível para a concorrência. Corresponde ao know-how de elementos individuais, que pode contribuir para a afirmação de competências-chave para o funcionamento da organização e constituir-se como o núcleo da MO a ser utilizado diariamente. No entanto é um know-how que pode estar "refém" de alguns elementos se estes não se disponibilizarem para o partilhar. Isto significa que o valor instrumental do conhecimento para a produção de ganhos de eficácia ou para a obtenção de vantagens competitivas da organização depende da intenção discricionária dos colaboradores partilharem ou não esse conhecimento e de o fazerem com mais ou menos pares e em maior ou menor grau.

A vantagem competitiva, obtida pela organização em consequência da aquisição, da posse e da partilha generalizada destes conhecimentos-críticos, pode perder-se se a gestão estiver centrada apenas numa gestão administrativa dos "recursos humanos", já que tudo se perderá quando estes abandonarem a organização - por uma de entre muitas razões comuns: reforma, rescisão contratual, mudanças na carreira, etc.. Neste caso, parte da memória da organização (a que está armazenada apenas nos indivíduos) pode ficar irremediavelmente perdida.

Um dos desafios atuais das organizações na construção e preservação da sua memória é a criação de mecanismos e processos que incentivem permanentemente a transferência de conhecimento entre os seus elementos, através da promoção de práticas de abertura, de manifestação de boa vontade, de disponibilidade e criação de oportunidades para a partilha. Num contexto altamente competitivo, em que se dão continuamente ganhos e perdas de pessoas, de resultados, de recursos, etc., a partilha de conhecimento parece ser uma atitude não "natural", que não se expressa com espontaneidade. Frequentemente os trabalhadores percebem que o seu conhecimento é um bem valioso, uma fonte de poder, pelo que podem não estar disponíveis para partilhar a fonte do seu poder (TEH; SUN, 2011), o que pode criar problemas sérios de sobrevivência à organização (LIN, 2007).

A resistência das pessoas ao desenvolvimento de práticas de colaboração e de partilha de experiências e conhecimentos no contexto da organização, assim como a propensão das pessoas para acumularem continuamente um volume crescente de conhecimentos (WAH, 2000), podem constituir-se como obstáculos significativos ao estabelecimento e à implementação de sistemas formais de gestão do conhecimento. O recurso a diferentes sistemas tecnológicos pode ser um elemento facilitador na identificação, construção, partilha, aplicação e preservação do conhecimento, mas estes por si só não garantem o sucesso dos processos de partilha. São as pessoas que os manipulam, pelo que o sucesso da regulação dos fluxos de comunicação depende da vontade das pessoas, ou seja, há um espaço de discricionariedade, que pode fazer toda a diferença.

No caso do conhecimento tácito (interno ao individuo), apenas o próprio pode decidir o que vai partilhar, com quem o vai partilhar, como vai partilhar e em que condições vai

Perspectivas em Gestão \& Conhecimento, João Pessoa, v. 8, n. 1, p. 3-19, jan./abr. 2018. 
continuar, ou não, a partilhar. Esta possibilidade real de opção coloca a partilha de conhecimento como um comportamento voluntário, de iniciativa pessoal, que não pode ser forçado e que depende também em larga medida de outras variáveis atitudinais, como, por exemplo, a confiança.

\section{COMPORTAMENTOS DE CIDADANIA ORGANIZACIONAL E PARTILHA DO CONHECIMENTO}

Os comportamentos voluntários dos colaboradores no local de trabalho têm sido estudados ao longo do tempo sob várias designações: espontaneidade organizacional (GEORGE; BRIEF, 1992), comportamentos extrapapel (VAN DYNE; CUMMINGS; PARKS, 1995) ou desempenho contextual (BORMAN; MOTOWIDLO, 1993). No entanto, o termo comportamentos de cidadania organizacional (ORGAN, 1988) é o que tem merecido mais atenção por parte dos investigadores.

O conceito de comportamento de cidadania organizacional (CCO), alicerçado nos conceitos de "vontade de cooperar" (BARNARD, 1938/1968) e de "comportamentos inovadores e espontâneos" (KATZ; KAHN, 1966/1978), definido inicialmente por Organ (1988, p. 4) como um "comportamento individual discricionário, não direta ou explicitamente reconhecido pelo sistema formal de recompensas e que no seu conjunto promove o eficaz funcionamento da organização", foi mais tarde redefinido como "atividades que apoiam o ambiente social e psicológico em que o desempenho das tarefas tem lugar" (ORGAN, 1997, p. 95) realçando a sua componente de não tarefa, que vai para além das funções rotineiras do posto de trabalho.

Embora não haja ainda um consenso sobre a estrutura dimensional do construto (NEVES; PAIXÃO, 2014), o modelo mais referido na literatura e mais utilizado em estudos sobre CCO é o modelo penta-fatorial de Organ (1988), onde os CCO são vistos e organizados em função da sua natureza, a saber: altruísmo (e.g. ajuda a pessoas específicas na organização), conscienciosidade (e.g. cumprimento de regras, para além do mínimo requerido na organização em assiduidade, observância de regras e regulamentos, gestão de pausas, etc.), desportivismo (e.g. comportamentos que resultam de uma vontade dos trabalhadores para tolerar, sem queixas, circunstâncias menos ideais), cortesia (e.g. comportamentos discricionários, com o objetivo de prevenir a ocorrência de problemas), virtude cívica (e.g. comportamento de participação responsável na vida da organização). Todos os comportamentos são positivos e de iniciativa individual, o que significa que não têm a sua origem em pressões ou coações de pares ou superiores hierárquicos e que de forma agregada contribuem para o incremento do desempenho da organização (ORGAN, 1988).

$\mathrm{Na}$ literatura académica portuguesa, a partilha do conhecimento foi considerada um CCO por Rego $(1999,2002)$, que a integrou numa dimensão que designou de harmonia interpessoal (e.g. ter espírito de camaradagem, partilhar conhecimentos e experiências). As outras dimensões de CCO identificadas pelo autor são espírito de iniciativa (e.g. apresentar soluções e não apenas problemas), conscienciosidade (e.g. trabalhar com rigor, mesmo na ausência de controlo), identificação com a organização (e.g. realizar esforço extra para benefício da organização).

Devido à natureza discricionária dos $\mathrm{CCO}$ e à elevada componente voluntariosa dos comportamentos de partilha do conhecimento, a relação entre os dois construtos tem sido avaliada, discutida e objeto de pesquisa de boas práticas de gestão de processos organizacionais (LIN, 2008; REZVAN; HOSSEIN; KOOHI, 2014). Por exemplo, Lin (2008) e Lin e Hsiao (2014) encontraram uma relação significativa entre todas as dimensões dos CCO e a partilha do conhecimento nas organizações. Teh e Sun (2011) consideram mesmo que os CCO são uma variável preditiva da partilha e conhecimento, atribuindo os baixos níveis de partilha

Perspectivas em Gestão \& Conhecimento, João Pessoa, v. 8, n. 1, p. 3-19, jan./abr. 2018. 
do conhecimento entre trabalhadores a baixos níveis de CCO (WASKO; TEIGLAND, 2004), ou seja, a partilha do conhecimento é influenciada pelos fatores antecedentes de CCO.

Todas as dimensões de CCO - altruísmo, cortesia, conscienciosidade, desportivismo e virtude cívica - têm uma influência significativa e simultânea na partilha do conhecimento (RAMASAMY; THAMARAISELVAN, 2011). Os colaboradores com elevados níveis de altruísmo ajudam os seus colegas e uma das formas como o fazem é providenciando o conhecimento especializado, nomeadamente, partilhando o conhecimento. Através da ajuda aos colegas partilham o seu conhecimento, contribuindo assim não só para a melhoria do desempenho e da competitividade da organização, mas também para transferir e preservar o conhecimento. O conhecimento deixa de ser apenas propriedade de um e passa a ser propriedade de vários elementos da equipa, o que aumenta a possibilidade deste não se perder num cenário de rotatividade laboral. Também os níveis de cortesia podem ter repercussões na partilha do conhecimento. Ter consciência de que partilhar o que se sabe evita problemas, pode ser o mote para a decisão de tomar a iniciativa de o fazer. O mesmo acontece com a conscienciosidade. Os indivíduos que evidenciam comportamentos de conscienciosidade são mais prováveis de partilhar conhecimento (principalmente tácito) uma vez que as fronteiras e os níveis de partilha deste tipo de conhecimento são muito dificilmente regulados por normas e regulamentos. $O$ desportivismo, que tem subjacente a abordagem das adversidades de forma positiva, pode levar a comportamentos de partilha de conhecimento individual para enfrentar e melhorar condições percebidas como indesejáveis. Do mesmo modo, as práticas de virtude cívica (envolvimento construtivo e responsável na vida da organização) implicam a partilha de conhecimento individual na geração de novas ideias ou soluções.

Também Rezvan, Hossein e Koohi (2014) identificaram uma relação entre práticas de gestão do conhecimento e todas as dimensões de CCO propostas por Organ (1988), concluindo que as pessoas que evidenciam elevados níveis de CCO em todas as suas dimensões são também mais passíveis de realizar práticas de gestão de conhecimento em todos os processos considerados: identificação, construção, expansão, partilha, aplicação e preservação do conhecimento.

O impacto positivo da realização dos CCO no desempenho profissional e organizacional tem vindo a ser estudado e evidenciado. Os CCO permitem antecipar significativamente a qualidade do desempenho profissional, uma vez que melhoram a habilidade dos trabalhadores e gestores de desempenharem as suas funções através de uma maior planificação, organização e resolução de problemas (BASU; PRADHAN; TEWARI, 2017 ; ORGAN; PODSAKOFF; MACKENZIE, 2006) e aumentam a probabilidade de partilha do conhecimento (LIN; HSIAO, 2014; TEH; SUN, 2011). Assim, importa perceber e realçar os fatores que podem estar na base ou constituírem-se como alicerces deste tipo de comportamentos.

A investigação sobre fatores antecedentes que incentivam os CCO tem evidenciado que os fatores disposicionais estão fracamente associados aos CCO (BORMAN et al., 2001). Embora alguns autores tenham posteriormente encontrado algumas ligações entre as necessidades de realização (BARUCH et al., 2004), a extroversão (OMAR; DELGADO, 2005) e a conscienciosidade (TAN; TAN, 2008), são as atitudes relacionadas com o trabalho que desempenham o papel mais relevante nas decisões individuais dos trabalhadores em realizar CCO (GUPTA; SINGH, 2017; ORGAN; PODSAKOFF; MACKENZIE, 2006). A tendência para a realização de $\mathrm{CCO}$ e a partilha do conhecimento está relacionada com atitudes como o envolvimento, a satisfação com o trabalho, o comprometimento organizacional (TEH; SUN, 2011), a perceção de justiça e a confiança no líder e na organização (LIN; HSIAO, 2014). Uma cultura de confiança é o fator mais importante na promoção do comportamento de partilha do conhecimento, influenciando significativamente a realização de CCO (GUPTA; SINGH, 2017). 


\section{Confiança e Partilha do Conhecimento}

Nas organizações, os grupos com baixos índices de confiança interpessoal reduzem a frequência das comunicações entre os seus membros, trocam menos informações entre si e partilham menos conhecimentos (ABRAMS et al., 2003; CHOW; CHAN, 2008; ZAND, 1972), enquanto, em situações novas e complexas, em que a incerteza e o risco predominam, os grupos com um elevado nível de confiança tendem a realizar mais esforços de coordenação das suas atividades, através da partilha de recursos e de conhecimentos, para enfrentarem os desafios colocados pela situação (GUPTA; SINGH, 2017; MCALLISTER, 1995; PORTO-GOMEZ et al., 2016; SMALIUKIENÉ et al., 2017). O que significa que a promoção de uma cultura organizacional baseada na confiança (CERDEIRA, 2010) tende a associar-se à partilha de conhecimento e à obtenção de ganhos de diversa ordem para as organizações. No setor público da economia, a confiança gera partilha de conhecimento e promove uma maior motivação dos colaboradores para a prestação de serviços públicos, assim como um comprometimento maior com os valores do bem comum (KIM, 2017), passando-se algo de semelhante também no relacionamento entre diferentes organizações, em que a confiança inter-organizacional se associa a uma maior partilha de conhecimento relevante para a determinação da qualidade da colaboração entre as partes (CHENG; YEH; TU, 2008).

No entanto, o conceito de confiança é um conceito complexo, que pode gerar entendimentos muito diversos quanto à sua caracterização e quanto à escolha da melhor forma de usar a confiança para promover boas práticas de partilha do conhecimento. Por exemplo, Alexopoulos e Buckley (2013), a partir da diferenciação da confiança pessoal e profissional em contexto organizacional, sugerem que a duração do relacionamento entre duas partes que partilham conhecimentos modela os efeitos da confiança profissional e da confiança pessoal na determinação da qualidade do conhecimento partilhado, embora em direções diferentes. Enquanto a confiança pessoal é mais importante na determinação da partilha de conhecimento no médio e longo prazo, a confiança profissional é mais relevante na partilha de conhecimento nas interações imediatas de curto prazo. No mesmo sentido, mas agora num contexto de coordenação de esforços num projeto de economia solidária numa comunidade rural, Silva et al. (2016) defendem que a confiança influencia as motivações das pessoas para a cooperação e para a partilha efetiva de informações e de conhecimentos. Com base num método qualitativo, estes autores sugerem ainda que confiança incentiva a realização de trocas recíprocas e que a frequência dessas trocas tende a associar-se a um processo de partilha crescente de informações e conhecimentos em relacionamentos sociais informais.

Sordi et al. (2014, p.129, p.130), a propósito do modo como os membros de uma cooperativa usam uma plataforma de gestão virtual de informação, por via da qual a organização formaliza as interações com o intuito das partes interessadas partilharem conhecimentos relevantes, conclui que a "confiança constitui um ingrediente fundamental para a cooperação entre os membros de uma equipa", ao mesmo tempo que é também um "fator primordial para o compartilhamento de conhecimentos".

Por sua vez, Chung e Jackson (2011), ao estudarem as condições em que a confiança pode estar associada à intensidade do processo de produção de conhecimento em equipas, concluíram que os membros de uma equipa de investigação (científica) produzem mais conhecimento quando são alvo da confiança dos outros membros da sua equipa, sendo esta associação mais forte quando os investigadores trabalham em tarefas com um elevado grau de interdependência em relação aos outros membros da equipa.

No campo específico da literatura de investigação publicada sobre a memória transativa, Ashleigh e Prichard (2012) defendem que a confiança é um determinante da memória transativa nas organizações e desenvolvem um modelo dinâmico sobre o papel

Perspectivas em Gestão \& Conhecimento, João Pessoa, v. 8, n. 1, p. 3-19, jan./abr. 2018. 
destes sistemas nas organizações. Tomando por referência as três componentes da perceção da confiabilidade propostas por Mayer, Davis e Schoorman (1995), aqueles autores sugerem a delimitação de vários estádios de desenvolvimento da memória transativa nas organizações. Defendem que estas etapas se desenvolvem em função do modo como os membros de uma equipa vão partilhando conhecimentos em consequência da experiência acumulada no desempenho de tarefas e da perceção da confiabilidade mútua que desenvolvem uns sobre os outros. Assim, cada estádio caracteriza-se por uma alteração no modo como cada membro da equipa avalia subjetivamente a confiabilidade dos seus parceiros ao longo de três dimensões: perceção das habilidades na execução de tarefas (perceção das competência), perceção das motivações para a realização dos objetivos da equipa (perceção das boa-vontade) e perceção do grau de cumprimento dos compromissos assumidos (perceção da integridade) de cada um dos membros da equipa (ASHLEIGH; PRICHARD, 2012). O que significa que a probabilidade das pessoas partilharem conhecimento organizacional depende não só da experiência acumulada de trabalho em equipa, mas sobretudo do quanto cada membro de uma equipa é percebido pelos pares como competente, como estando motivado para a realização dos objetivos comuns e como uma pessoa integra, ou seja, como uma pessoa percebida como confiável pelos pares.

\section{CONSIDERAÇões FinaIS}

As organizações que incentivam os CCO são locais de trabalho com maior atratividade para trabalhar (HUI; LEE; ROSSEAU, 2004) e são também as que conseguem contratar e reter os melhores trabalhadores (GEORGE; BATTENHAUSEN, 1990). Os CCO têm um papel estratégico para a realização dos objetivos mais complexos das organizações porque são capazes de inspirar os membros de uma organização a partilharem voluntariamente 0 conhecimento que acumularam pessoalmente a partir da experiência de execução das tarefas na organização. Sem a transmissão espontânea e informal deste conhecimento para os pares e para os novos membros ou até para os decisores da estrutura formal da organização, cometem-se e repetem-se erros vários, desperdiçam-se recursos e perdem-se vantagens competitivas importantes. Neste sentido, promover uma cultura de CCO baseada na confiança é criar condições propícias à partilha do conhecimento, é promover a passagem de conhecimento individual a conhecimento organizacional ou, no caso do conhecimento tácito, é promover a manutenção do conhecimento nos indivíduos que estão na organização, preservando assim a memória organizacional.

As melhores práticas de gestão de recursos humanos, que estão na base da promoção dos comportamentos e das atitudes dos colaboradores, são elementos relevantes na promoção de uma cultura de CCO, baseada na confiança mútua entre elementos das equipas e das organizações em geral e, consequentemente, na partilha do conhecimento.

As normas organizacionais que incentivam os comportamentos espontâneos propiciam a partilha do conhecimento (WASKO; TEIGLAND, 2004), pelo que a criação de uma cultura organizacional que encoraje os trabalhadores a preocuparem-se com o bem-estar de outros e da organização, levará com probabilidade à vontade de partilhar o conhecimento e consequentemente a preservar a memória organizacional.

Em síntese, considerando o quadro teórico exposto e os argumentos colhidos dos artigos revistos, sugerem-se algumas orientações estratégicas para a promoção de boas práticas de gestão do conhecimento e de construção da memória nas organizações.

Em primeiro lugar, é de chamar a atenção para o valor instrumental da experiência pessoal e subjetiva acumulada pelos colaboradores de uma organização. Ao fim de algum tempo, a reflexão pessoal produzida sobre as experiências idiossincráticas repetidas proporciona um conhecimento valioso sobre os erros a evitar, sobre a disponibilidade de

Perspectivas em Gestão \& Conhecimento, João Pessoa, v. 8, n. 1, p. 3-19, jan./abr. 2018. 
certas opções, sobre os critérios mais pertinemtes para optimizar a tomada de decisão, sobre a capacidade de antevisão de eventuais consequências de uma ação, sobre o sentido da evolução das dinâmicas de relacionamento, sobre a pertinência das oportunidades inesperadas, sobre o papel dos relacionamentos na resolução de problemas, etc. Esta reflexão pessoal sobre as vivências de trabalho na organização traduz um conhecimento tácito, o qual, apesar de ser muitas vezes injustamente desprezado pela circunstância da sua natureza ser quase inteiramente subjetiva, possui apesar de tudo um valor muito significativo. $E$ tão mais significativo, quanto é nele que por vezes as organizações constroem as suas vantagens competitivas frente à concorrência. Contudo, nem sempre os gestores de recursos humanos se dão conta deste ativo intangível, já que muitas vezes este conhecimento não é recolhido, não é analisado, nem sistematizado, sendo tão pouco avaliado e raramente objeto de transmissão, pelo que se perde quando quem o possui progride na carreira ou quando se reforma ou quando rescinde o seu contrato, etc.

Em segundo lugar, é importante salientar que o conhecimento tácito construído a partir de vivências pessoais apenas é partilhado em função da vontade própria de quem o produziu e o guarda. Muito embora as hierarquias e as estruturas formais de uma organizaçãoo possam apelar aos seus colaboradores para partilharem informações e conhecimentos de natureza pessoal, na realidade essa partilha apenas ocorre se as partes envolvidas confiarem mutuamente entre si. A generalidade dos estudos sobre os determinantes dos processos de partilha de informação ou de conhecimentos com valor (subjetivo ou mesmo objetivo) apontam no sentido de identificar a confiança interpessoal como um dos determinantes mais criticos para o incremento da cooperação, da colaboração ou para o incremento da generalidade dos processos de troca ou de partilha altruísta de recursos pessoais com terceiros. A construção de relacionamentos interpessoais (e profissionais) baseados na confiança e a construção de um clima ou de uma cultura organizacional baseada na confiança são assim prioridades a ter em consideração para aumentar a probabilidade de ocorrência de dinâmicas espontâneas de partilha de informaçãoo, de conhecimento e de recursos entre os pares ou entre a globalidade dos colaboradores de uma organização.

Por fim, o terceiro apontamento a valorizar desta reflexão remete para o argumento segundo o qual a promoçãoo de comportamentos de cidadania organizacional se puder constituir como uma ferramenta direta para a gestão do conhecimento organizacional e indirecta para a promoção de uma cultura de confiança capaz de gerar a partilha de informações e conhecimentos. Na medida em que remete para ações de natureza discricionária, voluntária e desinteressada, a partilha de conhecimentos acaba por ser a consequência natural e inevitável da vivência de práticas de cidadania nas organizações. Neste sentido, a gestão pode e deve gerir projectos e ações capazes de colocar a cidadania e a confiança no centro das suas prioridades, sobretudo se aspira a obter ganhos competitivos relevantes.

Como nota final, justifica-se recorrer novamente ao conceito de memória organizacional para evidenciar o quanto as temáticas de natureza fenomenológica, subjetiva e idiossincrática podem ganhar uma importância crescente na discussão sobre as políticas de gestão de processos organizacionais em empresas a operarem em mercados competitivos e globalizados cada vez mais baseados em economias do conhecimento.

\section{REFERÊNCIAS}

ABRAMS, L. C. et al. Nurturing interpersonal trust in knowledge-sharing networks. The Academy of Management Executive, v. 17, n. 4, p. 64-77, 2003.

Perspectivas em Gestão \& Conhecimento, João Pessoa, v. 8, n. 1, p. 3-19, jan./abr. 2018. 
ALEXOPOULOS, A. N.; BUCKLEY, F. What trust matters when: The temporal value of professional and personal trust for effective knowledge transfer. Group \& Organization Professional and Personal Trust for Effective Knowledge Transfer, v. 38, n. 3, p.361-391, 2013.

AMINU, I. M.; MAHMOOD, R. On the relationship between procedural and declarative organizational memory and their effects on SME performance. International Business Management, v. 10, n. 3, p. 241-247, 2016.

ARGOTE, L.; GUO, J. M. Routines and transactive memory systems: Creating, coordenating, retaining, and transfering knowledge in organizations. Research in Organizational Behavior, v.36, p. 65-84, 2016.

ASHLEIGH, M.; PRICHARD, J. An integrative model of the role of trust in transactive memory development. Group \& Organization Management, v. 37, n. 1, p. 5-35, 2012.

BARNARD, C. I. The functions of the executive. Cambridge: Harvard University Press, 1938/1968.

BARTOL, K. M.; SRIVASTAVA, A. Encoraging knowledge sharing. Journal of Leadership \& Organizational Studies, v. 9, n. 1, p. 64 - 76, 2002.

BARUCH, Y. et al. Prosocial behavior and job performance: does the need for control and the need for achievement make a difference? Social Behavior \& Personality, v. 32, p. 399-411, 2004.

BASU, E.; PRADHAN, R. K.; TEWARI, H. R. Impact of organizational citizenship behavior on Job Performance in Indian healthcare industries: The mediating role of socail capital. International Journal of Productivity and Performance Management, v. 66, n. 6, p.780-796, 2017.

BELL, S. J.; MENGUC, B. The employee-organization relationship, organizational citizenship behaviors, and superior service quality. Journal of Retailing, v. 78, n. 2, p. 131-146, 2002.

BIENSTOCK, C. C.; DEMORANVILLE, C. W.; SMITH, R. K. Organizational citizenship behavior and service quality. Journal of Services Marketing, v. 17, n. 4, p. 357-378, 2003.

BOCK, G. W.; KIM, Y. G. Breaking the myths of rewards: An exploratory study of attitudes about knowledge sharing. Information Resources Management Journal, v. 15, n. 2, p. 14-21, 2002.

BORMAN, W. C. et al. Personality predictors of citizenship performance. International Journal of Selection and Assessment, v. 9, p. 52-69, 2001.

BORMAN, W. C.; MOTOWIDLO, S. J. Expanting the criterion domain to include elements of contextual performance. In: SCHMITT, N.; BORMAN, W. C. Personal Selection in Organizations. San Francisco : Jossey-Bass, 1993. p. 71-98.

CARDOSO, L.; DUARTE GOMES, A.; REBELO, T. Gestão do conhecimento: Dos dados à informação e ao conhecimento. Comportamento Organizacional e Gestão, v. 9, n. 1, p. 55-84, 2003. 
CASCÃO, F. Gestão de competências do conhecimento e do talento: $O$ estado da arte da teoria e as melhores práticas na gestão das pessoas. Lisboa: Edições Sílabo, 2014.

CERDEIRA, J. P. O valor da confiança na definição da cultura organizacional. Exedra, v. 3, p. 125-134, 2010.

CHENG, J. H.; YEH, C. H.; TU, C. W. Trust and knowledge sharing in green supply chains. Supply Chain Management: An International Journal, v. 13, n. 4, p. 283-295, 2008.

CHOW, W. S.; CHAN, L. S. Social network, social trust and shared goals in organizational knowledge sharing. Information \& Management, v. 45, n. 7, p. 458-465, 2008.

CHUNG, Y.; JACKSON, S. E. Co-worker trust and knowledge creation: A multilevel analysis. Journal of Trust Research, v. 1, n. 1, p. 65-83, 2011.

DAVENPORT, T. H.; PRUSAK, L. Working knowledge. How organisations manages what they know. Boston: Harvard Business School Press, 1998.

GEORGE, J. M.; BATTENHAUSEN, K. Understanding prosocial behavior, sales performance, and turnover: A group-level analysis in a service context. Journal of Applied Psychology, v. 73, p. 698-709, 1990.

GEORGE, J. M.; BRIEF, A. P. Felling good- doing good: A conceptual analysis of the mood at work - organizational spontaneity relationship. Psychological Bulletin, v. 112, p. 310-329, 1992.

GUPTA, B.; SINGH, A. Predicting employee engagement, knowledge sharing \& OCB. The Indian Journal of Industrial Relations, v. 52, n. 4, p. 675-688, 2017.

HUANG, C.-C. et al. Mesurement of analytical knowledge-based corporate memory and its application. Decision Support Systems, v. 54, p. 846-857, 2013.

HUI, C.; LEE, C.; ROUSSEAU, D. M. Psychological contract and organizational citizenship behavior in China: Investigating generalizability and instrumentality. Journal of Applied Psychology, v. 89, n. 2, p. 311-321, 2004.

JIAO, C.; RICHARDS, D. A.; ZHANG, K. Leadership and organizational citizenship behavior: OCBspecific meanings as mediators. Journal of Business and Psychology, v. 26, n. 1, p. 11-25, 2011.

KATZ, D.; KAHN, R. L. The social psychology of organizations. New York: Wiley, 1966/1978.

KIM, S. Public service motivation, organizational social capital and knowledge sharing in the korean public sector. Public Performance \& Management Review, v. 41, n.1, p. 1-22, 2017.

LIN, C.-P. To share or not to share: Modeling knowledge sharing using exchange ideology as a moderador. Personnel Review, v. 36, n. 3, p. 457-475, 2007.

LIN, C.-P. Clarifying the relationship between organizational citizenship behaviors, gender, and knowledge sharing in workplace organizations in Taiwan. Journal of Business and Psychology, v. 22, p. 241-250, 2008. 
LIN, R. S.-J.; HSIAO, J.-K. The relationships between transformational leadership, knowledge sharing, trust and organizational citizenship behavior. International Journal of Innovation Management and Technology, v. 5, n. 3, p. 171-174, 2014.

MACKENZIE, S. B.; PODSAKOFF, P. M.; FETTER, R. Organizational citizenship behavior and objective productivity as determinants of managerial evaluations of salespersons' performance. Organizational Behavior and Human Decision Processes, v. 50, p. 123-150 1991.

MARQUES, D. V.; CARDOSO, L.; ZAPPALÁ, S. Knowledge sharing networks and performance. Comportamento Organizacional e Gestão, v. 14, n. 2, p.161-192, 2008.

MAYER, R. C.; DAVIS, J. H.; SCHOORMAN, F. D. An integrative model of organizational trust. Academy of Management Review, v. 20, n. 3, p.709-734, 1995.

MCALLISTER, D. J. Affect-and cognition-based trust as foundations for interpersonal cooperation in organizations. Academy of Management Journal, v. 38, n. 1, p. 24-59, 1995.

MOLINA, L. G.; VALENTIM, M. L. P. Memória organizacional como forma de preservação do conhecimento. Perpectivas em Gestão \& Conhecimento, v.5, n.2, p.147-169, 2015.

NASCIMENTO, N. M. et al. Gerenciamento dos fluxos de informação como requisito para a preservação da memória organizacional: Um diferencial competitivo. Perpectivas em Gestão \& Conhecimento, v. 6, Número especial, p. 29-44, 2016.

NEVES, P. C.; PAIXÃO, R. Comportamentos de cidadania organizacional: Uma revisão do conceito. Exedra, v. 9, p. 33-52, 2014.

NILAKANTA, S.; MILLER, L. L.; ZHU, D. Organizational memory management: Techonological and research issues. Journal of Database Management, v. 17, n. 1, p. 85-94, 2006.

NONAKA, I.; TAKEUCHI, H. The knowledge creating company: How japanese companies create the dynamics of innovation. New York: Oxford University Press, 1995.

OMAR, A.; DELGADO, H. U. Las dimensiones de personalidad como predictores de los comportamientos de ciudadanía organizacional. Estudos de Psicologia, v.10, p. 157-166, 2005.

ORGAN, D. W. Organizational citizenship behavior: the good soldier syndrome. Lexington MA: Lexington Books, 1988.

ORGAN, D. W. Organizational citizenship behavior: It's construct clean-up time. Human Performance, v.10, p. 85-97, 1997.

ORGAN, D. W.; PODSAKOFF, P. M.; MACKENZIE, S. B. Organizational citizenship behavior its nature, antecedents, and consequences. Thousand Oaks: Sage Publications, Inc, 2006.

PEREIRA, M. O. F. et al. Memória organizacional e suas contribuições para o fundo setorial -CTINFRA- UFPR. Perpectivas em Gestão \& Conhecimento, v. 6, n. 1, p. 128-140, 2016.

PORTO-GOMEZ, I.; OTEGI-OLASO, J. R.; ZABALA-ITURRIAGOGOITIA, J. Trust builders as open innovation intermediaries. Innovation: Management, Policy \& Practice, v. 18, n. 2, p. 145-163, 2016. 
PRAHALAD, C. K.; HAMEL, G. The core competence of the corporation. Harvard Business Review, May-June, p. 79-91, 1990.

RAMASAMY, M.; THAMARAISELVAN, N. knowledge sharing and organizational citizenship behavior. Knowledge and Process Management, v. 18, n. 4, p. 278-284, 2011.

REGO, A. Comportamentos de cidadania organizacional: Operacionalização de um construto. Psicologia, v. XIII, p. 127-148, 1999.

REGO, A. Comportamentos de cidadania nas organizações. Lisboa: McGraw-Hill, 2002.

REN, Y.; ARGOTE, L. Transactive memory systems 1985-2010: An integrative framework of key dimensions, antecedentes, and consequences. The Academy of Management Annals, v.5, n.1, p.189-229, 2011.

REN, Y.; CARLEY, K. M.; ARGOTE, L. The contigent effects of transactive memory: When is it more beneficial to know what others know? Management Science, v. 52, n. 5, p. 671-682, 2006.

REZVAN, N. S.; HOSSEIN, K.; KOOHI, N. M. Studying new views of organizational citizenship behaviors on knowledge management in executive systems of guilan province, Iran. International Journal of Engineering Research and Applications, v. 4, n. 6, p. 133-243, 2014.

SANTARÉM, V.; VITORIANO, M. C. D. C. P. Gestão da informação, fluxos informacionais e memoria organizacional como elementos da inteligência competitiva. Perspectivas em Gestão \& Conhecimento, v. 5, Número especial, p. 158-170, 2016.

SCHEIN, E. H. Organizational culture and leadership. 2. ed. San Francisco: Jossey- Bass, 1992.

SCHREIBER, D. O compartilhamento do conhecimento entre contratante e contratado no processo de externalização de atividades de P\&D. Perspectivas em Gestão \& Conhecimento, v. 5, n.2, p. 127-146, 2015.

SILVA, H. C. H.; BINOTTO, E.; VILPOUX, O. F. Cooperação e compartilhamento de informação entre atores sociais em um assentamento rural. Perspectivas em Gestão \& Conhecimento, v. 61, n.1, p. 98-108, 2016.

SMALIUKIENÉ, R. et al. Explicating the role of trust in knowledge sharing: A structural equation model test. Journal of Business Economics and Management, v. 18, n.4, p. 1-21, 2017.

SORDI, V. F.; BINOTTO, E.; RUVIARO, C. F. A cooperação e o compartilhamento de conhecimento em uma cooperativa de crédito. Pespectivas em Gestão \& Conhecimento, v. 4, n.1, p. 119-134, 2014.

SOUSA, A. J. F. P.; AMARAL, S. A. Impacto do compartilhamento da informação e do conhecimento para o desenvolvimento de inovações em grandes organizações. Pespectivas em Gestão \& Conhecimento, v. 2, Número especial, p. 12-26, 2012.

STEIN, E. W. Organizational memory: Review of concepts and recomendations for management. International Journal of Information Management, v.15, n. 2, p.17-32, 1995. 
TAN, H. H.; TAN, M. L. Organizational citizenship behavior and social loafing: The role of personality, motives, and contextual factors. The Journal of Psychology, v. 142, p. 89-108, 2008.

TEH, P.-L.; SUN, H. Knowledge sharing, job attitudes and organizational citizenship behavior. Industrial Management \& Data, v. 112, n. 1, p. 64-82, 2011.

TSAI, M.-S.; TSAI, M.-C. The influence of loyalty, participation and obedience on organizational citizenship behavior. International Journal of Business and Economic Affairs, v. 2, n.1, p. 6776, 2017.

VAN DYNE, L.; CUMMINGS, L. L.; PARKS, J. M. Extra-role behaviors: In pursuit of construct and definitional clarity (A bridge over muddied waters). In: STAW, B. M. Research in Organizational Behavior. Greenwich, Connecticut: JAI Press, v. 17, 1995. p. 215-285.

VAN HEIJST, G.; VAN DER SPEK, R.; KRUIZINGA, E. Corporate memories as a tool for knowledge management. Expert Systems With Applications, v. 13, n. 1, p. 41-54, 1997.

WAH, L. Making knowledge stick. In: CORTADA, J. W. The Knowledge management yearbook 2000-2001. Woburn, MA : Butterworth-Heinemann, 2000. p. 145-156.

WALSH, J. P.; UNGSON, G. R. Organizational memory. Academy of Management Review, v. 16, n. 1, p. 57-91, 1991.

WASKO, M. M.; TEIGLAND, R. Public goods or virtual commons? Applying theories of public goods, social dilemmas, and collective action to electronic networks of practice. The Journal of Information Technology Theory and Application, v. 6, n. 1, p. 25-41, 2004.

WEGNER, D. M. Transactive memory: A contemporary analysis of the group mind. In: MULLEN, B.; GOETHALS, G. R. Theories of group behavior. New York: Springer-Verlag, 1986. p. 185-208.

WEGNER, D. M.; GUILIANO, T.; HERTEL, P. T. Cognitive interdependence in close relationships. In: ICKES, W. J. Compatible and incompatible relationships. New York: Springer-Verlag, 1985. p. 253-276.

ZAND, D. E. Trust and managerial problem solving. Administrative Science Quarterly, v.17, n. 2, p. 229-239, 1972.

Artigo recebido em 05/01/2018 e aceito para publicação em 24/03/2018 